\title{
IMPACT OF OXYGEN CHEMISTRY ON THE EMISSION AND FLUORESCENCE SPECTROSCOPY OF LASER ABLATION PLUMES
}

\author{
K. C. Hartig ${ }^{1,2, *}$, B. E. Brumfield ${ }^{1}$, M. C. Phillips ${ }^{1}$, and S. S. Harilal ${ }^{1, *}$ \\ ${ }^{1}$ Pacific Northwest National Laboratory, Richland, WA 99352, U.S.A. \\ ${ }^{2}$ Nuclear Engineering Program, University of Florida, Gainesville, FL 32611, U.S.A. \\ *Corresponding Authors: kyle.hartig@ufl.edu; hari@pnnl.gov
}

\begin{abstract}
Oxygen present in the ambient gas medium may affect both laser-induced breakdown spectroscopy (LIBS) and laser-induced fluorescence (LIF) emission through a reduction of emission intensity and persistence. In this study, an evaluation is made on the role of oxygen in the ambient environment under atmospheric pressure conditions in LIBS and laser ablation (LA)-LIF emission. To generate plasmas, $1064 \mathrm{~nm}, 10 \mathrm{~ns}$ pulses were focused on an aluminum alloy sample. LIF was performed by frequency scanning a CW laser over the $396.15 \mathrm{~nm}\left(3 \mathrm{~s}^{2} 4 \mathrm{~s}{ }^{2} \mathrm{~S}_{1 / 2} \rightarrow 3 \mathrm{~s}^{2} 3 \mathrm{p}^{2} \mathrm{P}_{3 / 2}^{\circ}\right)$ Al I transition. Time-resolved emission and fluorescence signals were recorded to evaluate the variation in emission intensity caused by the presence of oxygen. The oxygen partial pressure $\left(p_{o}\right)$ in the atmospheric pressure environment using $\mathrm{N}_{2}$ as the makeup gas was varied from $0-400$ Torr $\mathrm{O}_{2}$. 2D-fluorescence spectroscopy images were obtained for various oxygen concentrations for simultaneous evaluation of the emission and excitation spectral features. Results showed that the presence of oxygen in the ambient environment reduces the persistence of the LIBS and LIF emission through an oxidation process that depletes the density of atomic species within the resulting laser-produced plasma (LPP) plume.
\end{abstract}

\section{KEYWORDS}

Laser ablation (LA); Laser induced fluorescence (LIF); Laser-induced breakdown spectroscopy (LIBS); Plasma chemistry; Combustion 


\section{INTRODUCTION}

Laser-induced breakdown spectroscopy (LIBS) is a well-known technique for elemental analysis [1]. Typically, LIBS is performed using a pulsed laser with high peak power to form a luminous micro-plasma on the surface of a target of interest. The generated plasma is short-lived but hot enough to generate excited ions and atoms which eventually emit radiation with characteristic frequency [2]. Currently LIBS is used in numerous fields ranging from environmental, medical, extra-terrestrial, archaeological, and to nuclear applications, for example [3-5]. There exist a large number of variables for LIBS optimization; however, the large parameter space is in fact very attractive for LIBS as an analytical technique [2, 6]. Some of the parameters that influence LIBS properties include laser wavelength, pulse width, intensity, focusing conditions, background or cover gas, detection timing (delay and gate width), etc. Among them, the composition and pressure of the ambient gas plays a principal role in the LIBS plume properties and therefore its analytical merits [7, 8]. Some of the well-known features of LIBS are in situ monitoring and hence rapid analysis, inherent standoff detection capability, and no requirement for sample preparation [9, 10]. However, even with such advantageous features, LIBS is not considered as an analytical tool for many applications because of its inferior detection limits and sample composition quantification difficulties compared to mass spectrometry-based techniques. Recently significant efforts have been made to improve the detection limits of LIBS which include, but are not limited to, double pulsing of the ablation laser, microwave enhanced LIBS, laser-induced fluorescence (LIF) of the laser ablation (LA) plumes, and spatial confinement of the expanding plasma plume $[2,11]$.

Among the various optical spectroscopic techniques (emission, absorption, and fluorescence), LIF and laser absorption spectroscopy (LAS) provide improved selectivity, sensitivity, and spectral resolution compared to traditional emission-based methods [12-19]. Hence LIF of LA plumes is capable of addressing some of the limitations in the figures of merit for LIBS measurements (precision, detection limits, line broadening, isotopic analysis, etc.) [20]. In LIF, atoms in the lower energy state for certain optical transitions in the plasma plume are excited preferentially by a second laser, and the emission resulting from the decay of the excited atoms is measured. Since LIF doesn't rely on the thermal excitation that is required for LIBS, the inherent linewidths of atomic transitions can be monitored when the plasma is cooler [21]. Moreover, the spectral resolution is dictated by the linewidth of the LIF pump source and hence this technique avoids the relatively large instrumental broadening encountered in LIBS.

The excitation spectrum, emission spectrum, and fluorescence lifetime or decay rates are the most common parameters of fluorescence spectroscopy for probing and sensing. Most of the previous studies related to LIF of LA plumes were performed using pulsed lasers that only provide a fixed wavelength resonance excitation $[16,22,23]$. Pulsed laser excitation is typically delayed for some time after the initial LA pulse and will lead to preferential excitation of the lower energy state population at a specific 
time in the plasma evolution; however, it is possible for LIF signal saturation to occur due to depletion of the lower energy state atoms [24]. It has to be mentioned that, similar to the population density of an excited state, the lower energy state of any transition is also defined by a Boltzmann distribution. As the plasma evolves and cools, the ground state population of an atomic species will increase, and continuous pumping using a CW laser may be beneficial to improve the signal intensity of LIF over the lifetime of the plasma instead of using pulsed laser excitation. Recently, we demonstrated the use of CW laser excitation to record 2D-fluorescence spectroscopy (2D-FS) in highly transient laser-produced plasmas (LPPs) by simultaneous measurement of the absorption laser wavelength and emission signal from coupled atomic transitions of $\mathrm{Al}$ in air [25]. 2D-FS of LA plumes provides excitation and emission spectra over a wide range of wavelengths and could be a versatile tool for isotopic analysis and for measurement of the inherent linewidths of atomic and molecular transitions.

Since emission and fluorescence signal intensities from LA plumes are particularly sensitive to various electronic energy transfer rates, significant variations in emission intensity can be expected with changes in the ambient gas pressure and composition [21]. Moreover, for any atomic spectroscopic tool, the persistence of the signal influences the analytical merits of the technique. For example, the cover gas used in the LIBS technique confines the plasma and hence maintains the temperature and density of the plasma system for a longer duration in comparison with LPP expansion under a rarefied atmosphere [26, 27]. It is well-known that oxygen present in the ambient environment may inhibit LIBS emission through plasma-assisted chemical reactions [7]. To avoid $\mathrm{O}_{2}$ effects, several LIBS schemes employ Ar as a cover gas [28]. As an inert gas, Ar removes any plasma chemistry considerations and improves the persistence of the LIBS emission. The effect of oxygen on LIF emission from LA plumes has not been studied extensively; however, in non-plasma conditions the presence of oxygen can result in reduction of atomic LIF emission intensity due to molecular oxide formation, collisional energy transfer, etc. Reduction of the LIF emission intensity and persistence through chemical reactions is currently applied in various applications ranging from discrimination in the detection of electron-deficient molecules, measuring the diffusion coefficient of oxygen in membranes, and live cell imaging [29].

All signals associated with LPPs, whether optical, electrical, or acoustic, are inherently transient and vary within the LPP due to the heterogeneous nature of the plasma. So, typically, the optimization of LPPs for various applications is done using an array of plasma diagnostic tools [30]. In this work, the role of oxygen in the measured LIBS and LIF signal is studied. Laser ablation was performed on an Al alloy sample in the presence of a cover gas at near atmospheric pressure conditions with a number of oxygen concentrations. To perform LIF, an ultra-narrowband tunable excitation source was used. LIF excitation increased the magnitude and persistence of emission from selected atomic transitions. The temporal emission features of both LIBS and LIF emission are monitored and compared. Our results 
show that the presence of a reactive species like oxygen within the ambient environment causes plasmaassisted chemical reactions to occur, which acts to deplete the atomic species present in the expanding plasma plume and significantly reduces the persistence of the LIBS and LIF emission.

\section{EXPERIMENTAL DETAILS}

Figure 1 gives a simple schematic of the experimental set up. The plasmas were produced on an Al 6061 alloy target by focusing the fundamental radiation $(1064 \mathrm{~nm})$ from a Q-switched Nd:YAG laser with a pulse width of $\sim 10 \mathrm{~ns}$ (full width half maximum, FWHM) and a repetition rate of $10 \mathrm{~Hz}$ onto the surface of the sample. The laser energy at the target surface was attenuated by a combination of a half-waveplate and a cube polarizer. The laser pulse energy inside the vacuum chamber after attenuation and reflection off of the optical window was determined to be $22 \mathrm{~mJ}$, which resulted in a power density at the target of 8 GW.cm ${ }^{-2}$ for a spot size of $\sim 250 \mu \mathrm{m}$. The target was positioned on an $\mathrm{x}-\mathrm{y}-\mathrm{z}$ translation stage which was moved to avoid target cratering effects as well as to align the excitation beam with respect to the plasma plume. The laser ablation was performed in a vacuum chamber that has ports for pump and excitation laser entry, collection of light, pressure gauges, and other diagnostic instrumentation along with electrical and gas feed-thru. The vacuum chamber is capable of being pumped down to rarefied pressures of $<10^{-6}$ Torr. The oxygen concentration was varied by adjustment of the $\mathrm{O}_{2}$ partial pressure $\left(p_{o}\right)$ present in the atmospheric pressure $\mathrm{N}_{2}$ ambient environment within the vacuum chamber. The vacuum chamber was pumped down using the turbo-molecular pump to $\sim 10^{-4}$ Torr residual pressure between each change in $\mathrm{O}_{2}$ to reduce any possible contamination between each change in the atmospheric composition.

A continuous wave (cw), ultra-narrowband ( 100 kHz) frequency doubled Ti:Sapphire laser (M Squared Lasers SolsTiS ECD-X) was used to excite the Al I $396.15 \mathrm{~nm}$ lower energy state population in the plasma for LIF measurements, which provides $\mathrm{cw}$ power levels $\sim 350 \mathrm{~mW}$. The tunable $\mathrm{cw}$ laser is capable of being scanned across the Al I transitions with a maximum scan range of $\sim 24 \mathrm{pm}$. The LIF excitation laser was focused using a $\mathrm{f}=40 \mathrm{~cm}$ lens with a spot size of $\sim 100 \mu \mathrm{m}$ inside the plasma and intercepted the plasma plume parallel to the target surface at a distance $1 \mathrm{~mm}$ from the target surface. The wavelength of the excitation laser was continuously monitored using a wavelength meter. For LIBS measurements, the tunable cw LIF excitation laser was blocked utilizing a beam block before the vacuum chamber. 


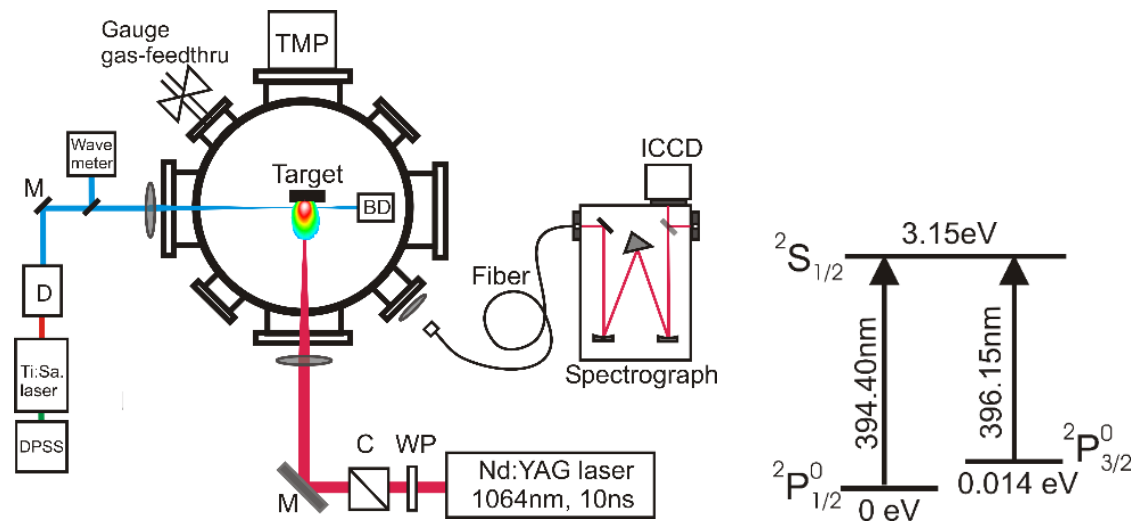

Figure 1. (left) Simplified schematic of the experimental setup and (right) partial Al I energy diagram. The Al I $396.15 \mathrm{~nm}$ transition was pumped for LIF in this work and both the 394.40 and $396.15 \mathrm{~nm}$ emission lines were monitored. BD - beam dump, C - cube polarizer, D - CW frequency doubler, DPSS - pump laser, ICCD intensified charge-coupled device, $\mathrm{M}$ - mirror, TMP - turbo-molecular vacuum pump, WP - half waveplate.

To collect LIBS and LIF signals, a plano-convex lens with focal length of $15 \mathrm{~cm}$ and aperture of $5 \mathrm{~cm}$ was positioned at a $\sim 45$-degree angle with respect to the target normal. The collected light was focused onto the tip of a $400 \mu \mathrm{m}$ multimode fiber optic cable, which transported the light to a $0.5 \mathrm{~m}$ spectrograph. All measurements were conducted in a spatially-integrated manner. The spectrograph system consists of three gratings $(600,1200$, and 2400 grooves/mm) and an intensified charge-coupled device (ICCD). All spectra were background-subtracted and an entrance slit width of $50 \mu \mathrm{m}$ was used. 2D-FS images were generated by measuring the LIF emission at $394.40 \mathrm{~nm}$ and $396.15 \mathrm{~nm}$ as the cw laser was scanned across the Al I $396.15 \mathrm{~nm}$ transition. Within this work the measured emission signal when the probe laser is incident on the plasma will be referred to as the measured LIF emission signal.

\section{RESULTS}

Most of the previous studies on LIF of LA plumes used a pulsed laser as an excitation source [16, 19, 22, 31]. In the present study, we used a cw laser as a LIF excitation source which provides unique advantages, such as higher spectral resolution, constant re-excitation during the entire lifecycle of the plume, and simpler parametric optimization (no inter-pulse delay, for example). The role of $\mathrm{O}_{2}$ on LIBS and LIF signals are monitored by addition of $100-400$ Torr $p_{o}$ to $\mathrm{N}_{2}$ while maintaining the total ambient pressure at near atmospheric levels (700 Torr). Since the mass differences between the $\mathrm{N}_{2}$ and $\mathrm{O}_{2}$ molecules are not significant, plume confinement is expected to be more or less similar at various gas mixture levels used.

\section{IIIa. LIBS and LIF of Al in an ambient $N_{2}$ atmosphere}


In the LIF technique, a probe beam re-excites atoms of the analyte of interest in the expanding plasma plume. This technique is particularly useful for analysis of high- $\mathrm{Z}$ materials, which exhibit congested emission spectra [21, 22, 32]. The Al I $396.15 \mathrm{~nm}$ transition $\left(3 \mathrm{~s}^{2} 4 \mathrm{~s}^{2} \mathrm{~S}_{1 / 2} \rightarrow 3 \mathrm{~s}^{2} 3 \mathrm{p}^{2} \mathrm{P}_{3 / 2}^{\circ}\right)$ was used for LIF excitation in this study, while the Al I $394.40 \mathrm{~nm}$ emission line $\left(3 \mathrm{~s}^{2} 4 \mathrm{~s}^{2} \mathrm{~S}_{1 / 2} \rightarrow 3 \mathrm{~s}^{2} 3 \mathrm{p}^{2} \mathrm{P}_{1 / 2}^{\circ}\right)$, which shares the same upper energy level (see Fig. 1(a)), was monitored to generate the resulting LIBS and LIF spectra [33]. Figure 2 shows the temporal evolution of the LIBS and LIF emission spectra using a $2 \mu$ sate width and $2 \mu$ s gate delay step under an ambient $\mathrm{N}_{2}$ atmosphere. For this figure, the LIF excitation laser was resonant with the Al I $396.15 \mathrm{~nm}$ transition, and it clearly shows that LIF emission is produced over significantly longer times than the LIBS emission due to the continuous pumping of the lower energy state of the Al I $396.15 \mathrm{~nm}$ transition [25].

Figure 3 shows the peak intensity of the data presented in Fig. 2 for both the LIBS and LIF spectra. An exponential intensity decay $\left(I(t)=I_{0}+A e^{-t / t_{0}}\right)$ curve was applied to two different temporal regimes present in the LIBS data shown in Fig. 3. The LIBS emission rapidly decreases at early times (3 $50 \mu \mathrm{s})$ with $\mathrm{t}_{0}=6.8 \mu \mathrm{s}$ as the LPP supersonically expands and cools. At later times $(50-200 \mu \mathrm{s})$, the emission decreases at a much lower rate of $t_{0}=48 \mu$ s due to regulation of the temperature and density as a result of confinement by the ambient atmosphere. The intensity of the LIF emission in the first $\sim 20 \mu$ s is not significantly enhanced due to the high temperature of the plasma and therefore limited lower energy state population; however, at later times the LIF emission is vastly enhanced compared to the LIBS emission. When the measured LIF emission intensity is divided by the measured LIBS emission intensity, the ratio is always greater than unity (shown in the inset of Fig. 3). The ratio indicates, even at early times ( $₹ 25 \mu \mathrm{s}$ ), significant LIF emission exists in addition to the thermally excited LIBS emission. For the LIF emission a second peak is observed at $\sim 325 \mu \mathrm{s}$, shortly before the emission begins to decrease. The LIF emission measurement was terminated at $400 \mu \mathrm{s}$; however, the LIF signal can be observed at much later times if the acquisition gate width is increased, since a relatively short $(2 \mu \mathrm{s})$ gate width was used to generate the temporal measurements presented here. 

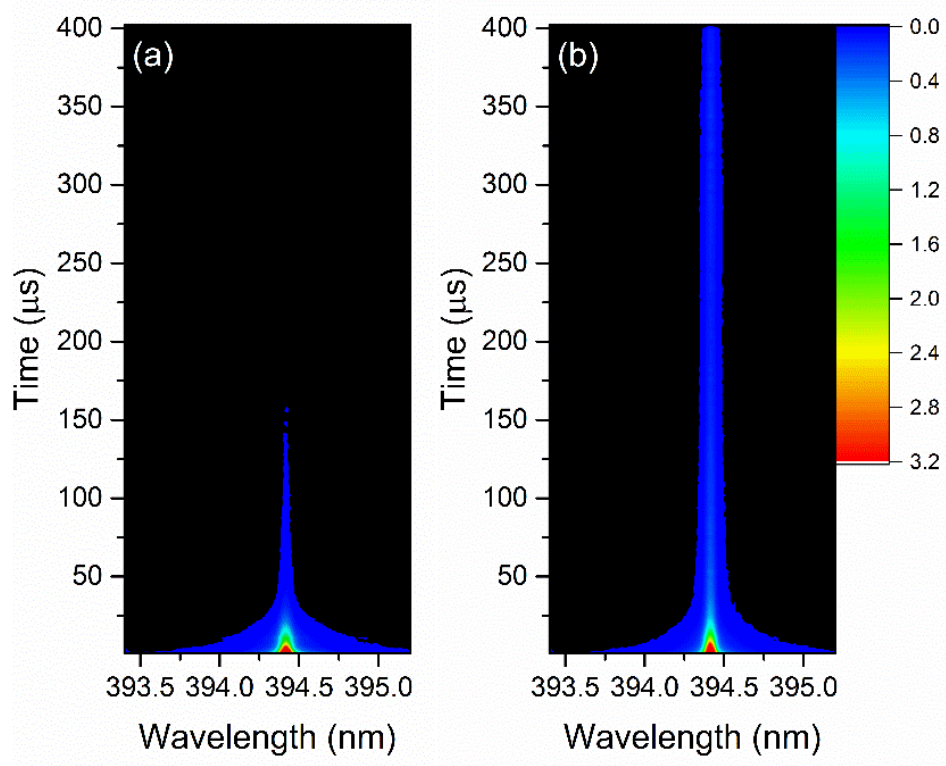

Figure 2. Temporal evolution of the (a) LIBS and (b) LIF Al I $394.40 \mathrm{~nm}$ emission line under an ambient 700 Torr $\mathrm{N}_{2}$ atmosphere. A $2 \mu$ s gate width was used with constant $2 \mu$ s gate steps to acquire the temporal evolution of the Al I emission line.

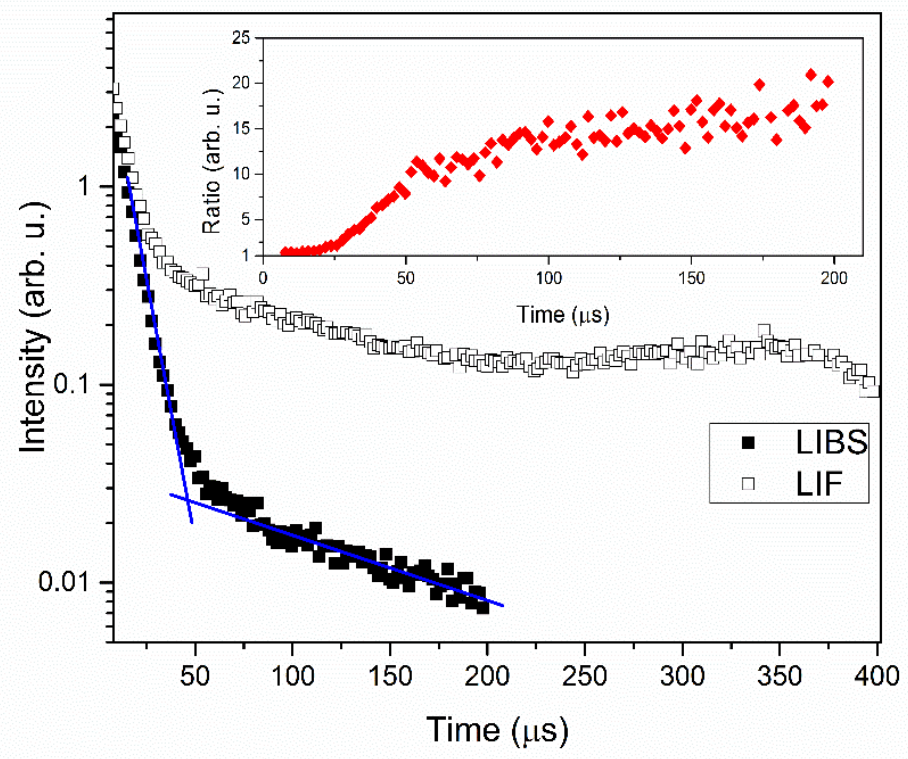

Figure 3. Peak intensity of the $\mathrm{N}_{2}$ LIBS (filled squares) and LIF (open squares) signal for the data shown in Fig. 2. Two blue lines have been added to the LIBS curve to separate out the two different temporal regimes observed in the decay of the LIBS emission intensity. The LIF emission intensity was divided by the LIBS emission intensity for each time step and is shown in the inset (red diamonds).

\section{IIIb. The impact of $\mathrm{O}_{2}$ ambient on LIBS and LIF signals}


Many measurements require LA to occur under ambient atmospheric conditions; therefore, the effect that atmospheric composition has on the resulting LPP and the associated LIBS and LIF signals is extremely important. The presence of oxygen in the ambient atmosphere has been shown to react with atomic species in the plasma plume to form molecular radicals through combustion or oxidation processes [34]. For $\mathrm{Al}$, the formation of $\mathrm{AlO}$ within the plasma readily occurs under ambient air atmospheric conditions through combustion with $\mathrm{O}_{2}$ in the atmosphere $[35,36]$. In order to elucidate the effect that the oxidation process has on the observed LIBS and LIF emission signals, the partial pressure of $\mathrm{O}_{2}$ in the vacuum chamber was varied from 100 to 400 Torr and $\mathrm{N}_{2}$ was added to maintain a near ambient atmospheric pressure of 700 Torr within the chamber. Use of a constant pressure reduces the effect that changes in plasma confinement might have on the measured emission signals.
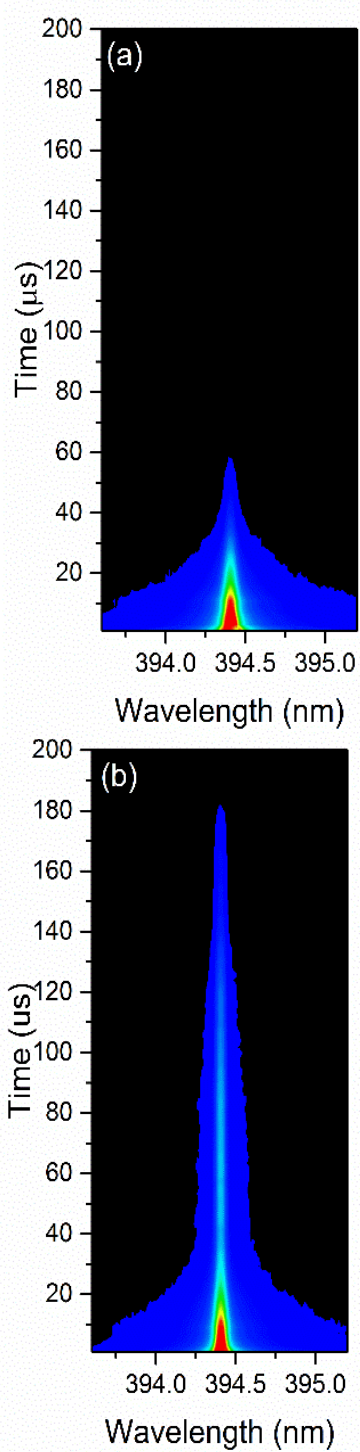

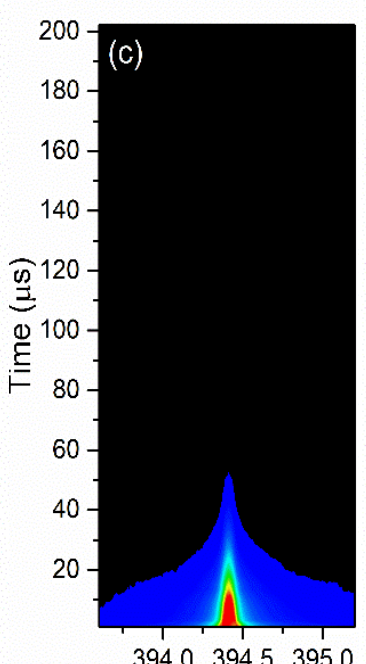

394.0394 .5395 .0

Wavelength $(\mathrm{nm})$

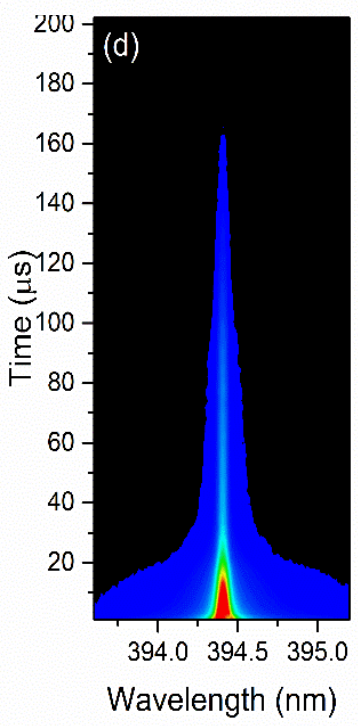

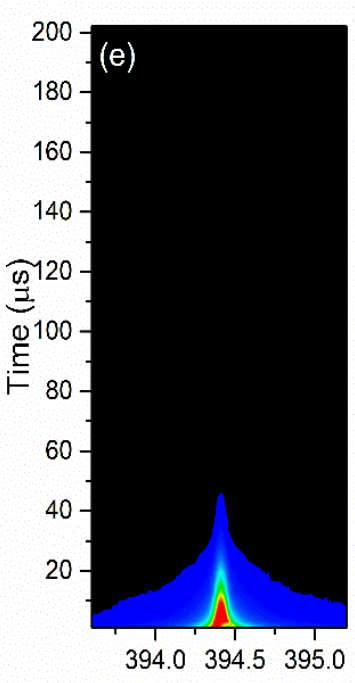

Wavelength $(\mathrm{nm})$

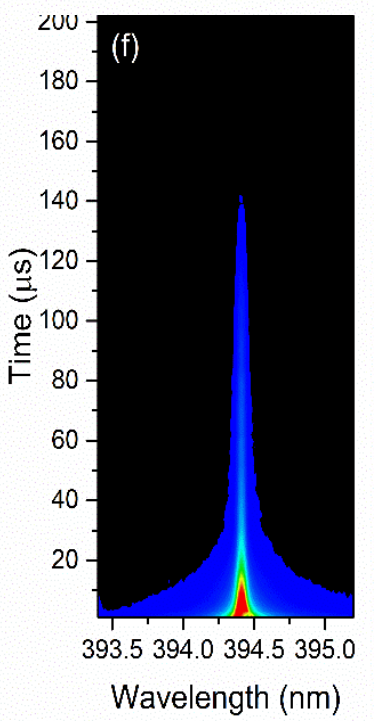

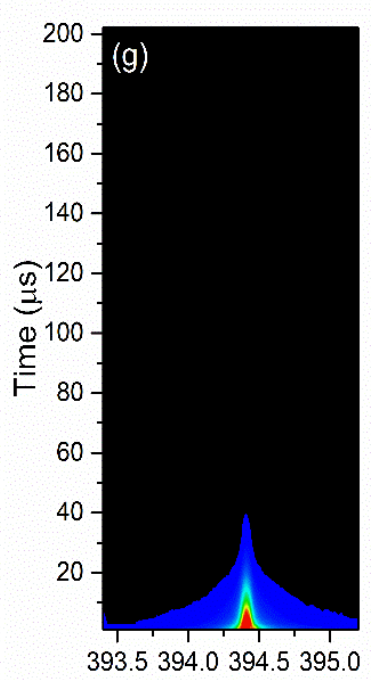

Wavelength $(\mathrm{nm})$

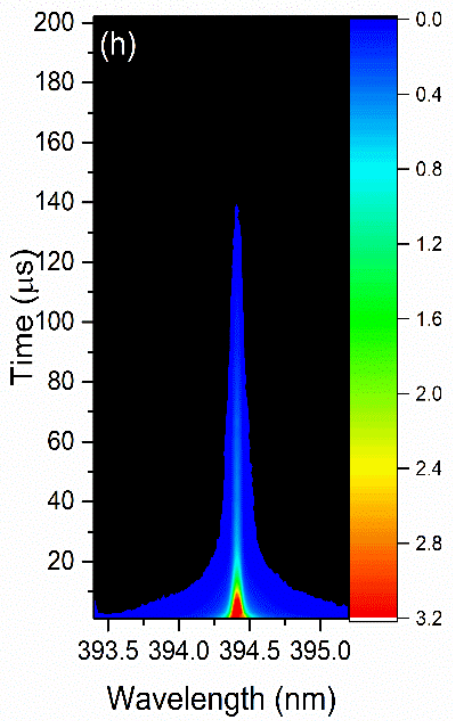

Figure 4. Temporal evolution of the emission signal for (a) 100 Torr $p_{o}$ LIBS \& (b) LIF, (c) 200 Torr $p_{o}$ LIBS \& (d) LIF, (e) 300 Torr $p_{o}$ LIBS \& (f) LIF, and (g) 400 Torr $p_{o}$ LIBS \& (h) LIF. Acquisition settings were the same as reported in Fig. 2. All images employ the same intensity scale. 
Figure 4 shows the temporal evolution of the LIBS and LIF emission signal as the concentration of $\mathrm{O}_{2}$ is varied within the chamber. As the concentration of $\mathrm{O}_{2}$ in the environment was increased the LIBS emission persistence gradually decreased, as shown in Fig. 4(a, c, e, \& g). A similar gradual decrease is observed for the LIF emission persistence, shown in Fig. 4(b, d, f, \& h), as the $\mathrm{O}_{2}$ concentration was increased. The peak intensity temporal evolution of the LIBS and LIF emission data shown in Fig. 4 is shown in Fig. 5. For comparison purpose, the temporal evolution of LIBS and LIF in 700 Torr $\mathrm{N}_{2}$ is also added in Fig. 5. In Fig. 5 the first $8 \mu$ s of the measured signal is saturated due to the requirement that constant acquisition parameters be used over the entire temporal evolution of the LPP; therefore, the peak intensity at early times is not able to be compared under the different environmental conditions.

The gradual reduction in the LIBS emission persistence is clearly seen in Fig. 5(a) as the oxygen concentration in the ambient environment is increased, and the emission intensity in each case decreased by three orders of magnitude over $\sim 75 \mu$ s before the signal is no longer detectable. The LIF signal shows obvious temporal features when $\mathrm{O}_{2}$ is present in the ambient environment, as shown in Fig. 5(b). LIF emission is found to be enhanced with an increase in $p_{o}$ at times $\widetilde{<} 125 \mu \mathrm{s}$, with an appearance of a secondary maxima. However, similar to LIBS, the persistence of LIF is also considerably reduced with increased $p_{o}$. As the concentration of $\mathrm{O}_{2}$ is increased, the secondary peak continues to move earlier in time and the lifetime is reduced further.
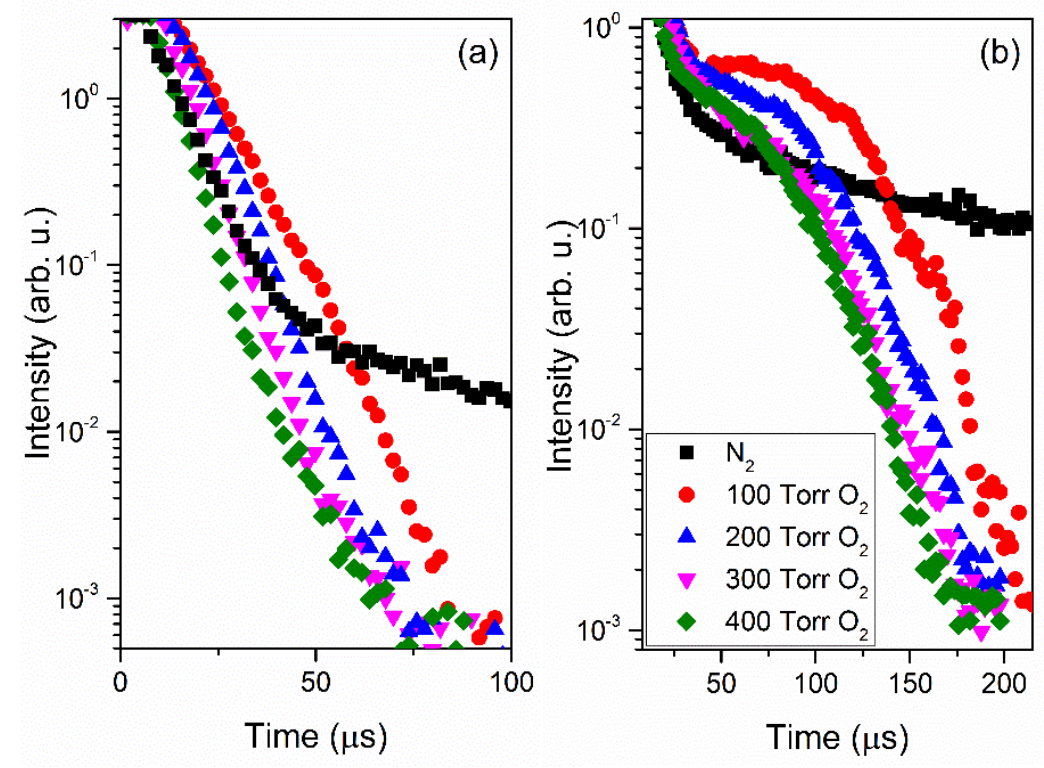

Figure 5. Temporal evolution of the peak intensity for the (a) LIBS emission and (b) LIF emission shown in Figs. 2 $\& 4$.

\section{DISCUSSION}


The present results clearly show that the addition of oxygen to the ambient environment influences the persistence of both LIBS and LIF emission. The persistence is found to decrease as the concentration of $\mathrm{O}_{2}$ is increased from 100 to 400 Torr $p_{o}$, due to a reduction in both the excited and lower energy state populations of the studied transition. The intensity of an emitting transition in thermal equilibrium can be expressed as [37]

$$
I_{2 \rightarrow 1}=\left(\frac{h v}{4 \pi}\right) A_{21} g_{2}\left(n_{a} / U_{a}\right) e^{-E_{2} / k T_{e x c}}
$$

where 2 and 1 represent the upper and lower energy levels; $\mathrm{A}_{21}$, the transition probability; $\mathrm{g}_{2}$, the degeneracy of the upper energy level; $n_{a}$, atom density; $U_{a}$, the partition function; $E_{2}$, energy of the upper energy level; $\mathrm{k}$, the Boltzmann constant; and $\mathrm{T}_{\mathrm{exc}}$, the excitation temperature, respectively. According to Eqn. 1, the persistence of LIBS emission is determined by the time dependence of thermal excitation and number density of the atoms in the plasma plume. As the plume expands into an ambient environment, the plasma begins to cool and the measured LIBS emission intensity commensurately decreases.

If reactive species are present in the ambient environment, plasma-assisted chemical reactions can occur, which will deplete the population density of excited and lower energy state atoms in the plasma plume [27, 38, 39]. In the present experiment, $\mathrm{N}_{2}$ and $\mathrm{O}_{2}$ are used (in varying relative concentrations for a total pressure of 700 Torr) as the buffer gases. By comparison of the time evolution of the LIBS and LIF emission intensity in atmospheres where $\mathrm{O}_{2}$ was present, a reduction in both the LIBS and LIF persistence was observed for increasing $\mathrm{O}_{2}$ concentration; therefore, it can be concluded that oxidation in the plume is prevalent compared to nitride formation. Previous studies also showed the predominance of AlO emission when $\mathrm{Al}$ plasma interacted with ambient air, which is consistent with the standard heat of formation being strongly exothermic for $\mathrm{AlO}$ and strongly endothermic for AlN [40]. During Al combustion, other molecules such as $\mathrm{Al}_{2} \mathrm{O}$ and $\mathrm{AlO}_{2}$ can also be formed as intermediates, but they do not have strong emission bands in the visible region and are therefore not observed in this experiment [41].

Figure 3 showed that the temporal decay of LIBS possesses multiple regimes: the signal drops rapidly at early times followed by much slower decay at later times. Since the difference in molecular masses of $\mathrm{N}_{2}$ and $\mathrm{O}_{2}\left(\mathrm{~N}_{2, \mathrm{~m}}=28 \mathrm{amu}\right.$ and $\left.\mathrm{O}_{2, \mathrm{~m}}=32 \mathrm{amu}\right)$ is not significant and considering the constant ambient pressure used (700 Torr) in our studies, the mass density of the shock layers is anticipated to be more or less the same as the concentration of $\mathrm{O}_{2}$ is varied in the ambient environment. Previous studies employing computational fluid dynamics (CFD) modeling showed that shock pressures can reach $\sim 100$ $\mathrm{MPa}$ at early times of plume expansion under atmospheric conditions and decay to $\sim 1 \mathrm{MPa}$ within $2 \mu \mathrm{s}$ [42]. The rapid temporal decay of LIBS emission at early times regardless of the composition of the ambient medium $\left(\mathrm{N}_{2} p_{N}+\mathrm{O}_{2} p_{o}\right)$ could be due to strong shock wave expansion, which in turn allows rapid expansion of the plasma in the rarefied regime created by the shock waves and limited interaction 
between the plasma plume and ambient gas. As time evolves the shock waves become weaker, along with a reduction in propagation velocity. Previous studies also showed that the position of the shock front (R)

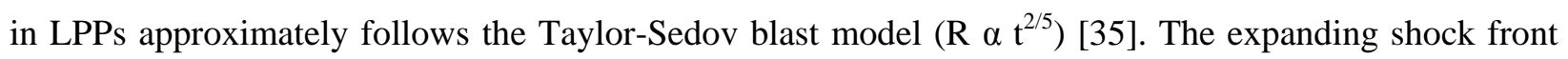
eventually stops and collapses when the shock pressure equilibrates with ambient pressure ( 0.1 MPa), and at this stage diffusion of ambient molecules into the plasma plume will be prevalent.

When $\mathrm{O}_{2}$ is present in the environment, $\mathrm{Al}$ will react through a combustion process to form $\mathrm{AlO}$ and additional polyatomic oxides like $\mathrm{AlO}_{2}, \mathrm{Al}_{2} \mathrm{O}_{2}$, and $\mathrm{Al}_{2} \mathrm{O}_{3}$ [7, 43]. The favored temperatures for the formation of $\mathrm{AlO}$ are $\sim 3000-6000 \mathrm{~K}[35,44]$. Our recent studies have shown that plume hydrodynamics play a significant role in defining plasma chemistry and molecular formation. Al oxidation can be confirmed through observation of the AlO B-X bands. Time-resolved studies of AlO showed that its emission is predominant in the temporal window of $10-60 \mu \mathrm{s}$. The recorded AlO $\Delta v=-1$ emission bands between 507 and $516 \mathrm{~nm}$ for various $\mathrm{O}_{2}$ partial pressures are given in Fig. 6(a). The AlO spectra were measured with a $10 \mu$ s gate delay and over a large gate width $(50 \mu \mathrm{s})$; therefore, the AlO spectra shown in Fig. 6(a) are time averaged. As expected, the AlO emission intensity is lowest for the lowest of the $\mathrm{O}_{2}$ concentrations used ( 100 Torr $p_{o}$ ). As the $\mathrm{O}_{2}$ concentration is increased, the $\mathrm{AlO}$ emission begins to increase up to 300 Torr $p_{0}$ and subsequently begins to decrease at higher $\mathrm{O}_{2}$ partial pressures. The decrease in the $\mathrm{AlO}$ emission intensity observed for the highest oxygen concentration could be due to the formation of more complex polyatomic oxides. Although there have been a number of works on the molecular formation and emission mechanisms in LPPs, the specific reaction pathways and kinetic rates associated with Al plasma chemistry are not specifically known [35].

Based on Eqn. 1, the emission features of a LPP plume is determined by the temperature and number density of atoms in the plume. So, the molecular temperatures of $\mathrm{AlO}$ were measured using PGOPHER spectral fitting [45-47]. The molecular temperature determined by the spectral analysis describes the rotational and vibrational temperature for AlO, which are based on the assumption of local thermodynamic equilibrium (LTE). For simulation and fitting of the molecular spectra, only emission from transitions in the $\Delta v=-1$ band are considered. Figures 6(b) and 7 show an example of the spectral model output by the PGOPHER program and the measured molecular temperatures, respectively. As the concentration of $\mathrm{O}_{2}$ was increased the AlO molecular temperature decreased, as shown in Fig. 7. The molecular temperature determined in this work agrees well with previous work available in the literature [7]. 

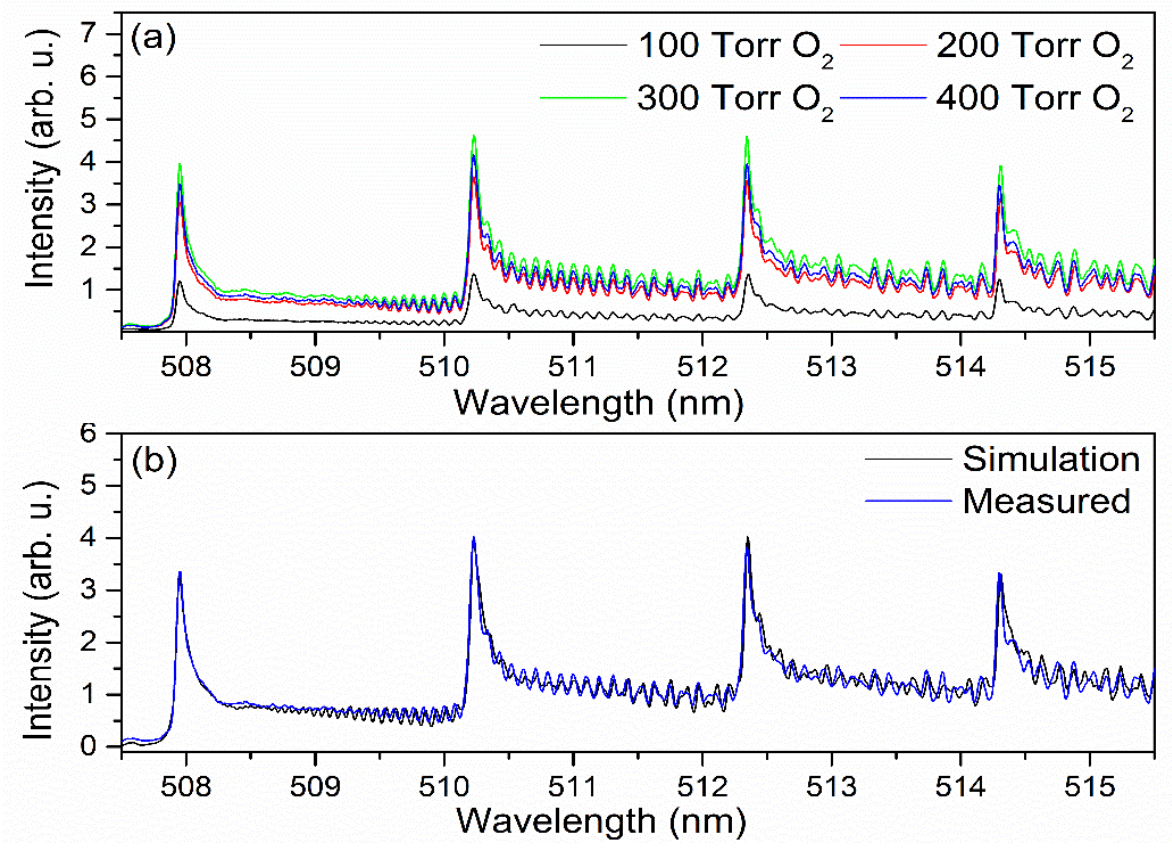

Figure 6. (a) Measured AlO spectra for various $p_{o}$ and makeup $\mathrm{N}_{2}$ added to maintain constant 700 Torr total pressure. Spectra were acquired with a $10 \mu$ s gate delay and $50 \mu$ s gate width and were corrected for intensity using a DH2000 calibration lamp. (b) PGOPHER simulation (black) of the measured 400 Torr $p_{o}$ AlO spectrum (blue) shown in Fig. 6(a) for comparison.

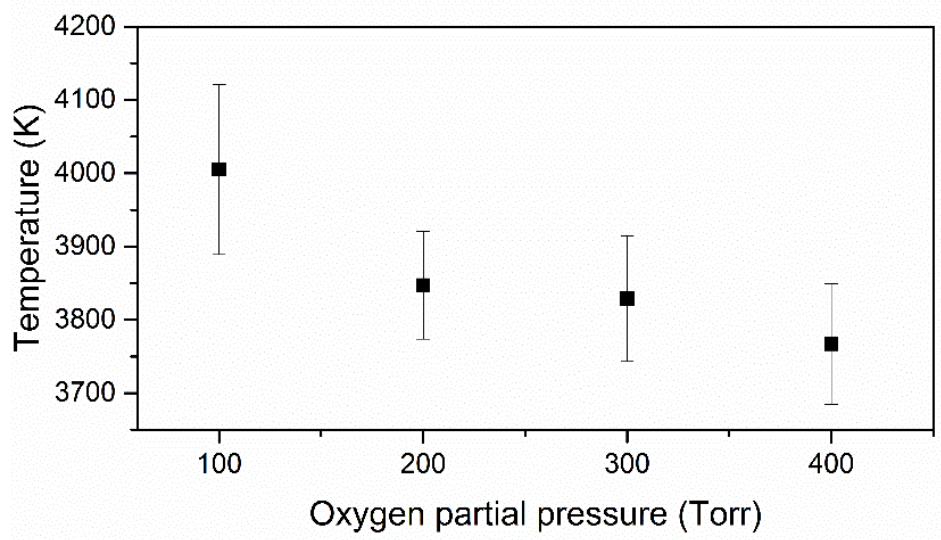

Figure 7. Molecular temperature derived from spectral analysis of the experimental data using the PGOPHER molecular analysis program for each oxygen concentration given in the figure above. Error bars represent a 3-sigma error determined from the least squares fitting routine.

Limited studies exist on the role of oxygen on LIBS emission. Piehler et al. [7] compared AlO emission in air and oxygen ambient and found that $\mathrm{AlO}$ emission appeared earlier in oxygen ambient compared to air, concluding that the formation of $\mathrm{AlO}$ was through the reaction of ground state $\mathrm{Al}$ with ambient oxygen $\left(\mathrm{Al}(\mathrm{g})+\mathrm{O}_{2} \rightarrow \mathrm{AlO}+\mathrm{O}\right)$. However, our results showed that $\mathrm{AlO}$ persistence is short lived ( $\widetilde{<} 60 \mu \mathrm{s})$ compared to LIF emission ( $\widetilde{>} 150 \mu \mathrm{s})$. Buckley [48] reported that the addition of $\mathrm{O}_{2}$ to the ambient gas has a large effect on the LIBS signal levels of $\mathrm{Pb}$ and the reduction in signal with the addition 
of oxygen followed approximately $2.5 \%$ decrease in signal for every $1 \%$ increase in $\mathrm{O}_{2}$ concentration. However, their results also showed different signal trends for $\mathrm{Cr}$ and $\mathrm{Be}$ line emissions in comparison with $\mathrm{Pb}$. Gleason and Hahn [49] reported a dramatic reduction in LIBS signal from a $\mathrm{Hg}$ plasma in air in comparison with $\mathrm{N}_{2}$, which is similar to the results observed in a $\mathrm{Pb}$ plasma by Buckley [48]. These previous results highlight that the $\mathrm{O}_{2}$-initiated plasma chemistry and its associated reduction of the excited state population depends strongly on the target material and its matrix.

In comparison with LIBS, the LIF emission peaked at later times and persisted longer. At early times, the magnitude of the emission signal (Fig. 5b) is a result of both thermally-excited atoms (LIBS) and optical pumping of atoms to an excited state (LIF) within the plume. The optimal conditions for LIF corresponds to the presence of a cooler atomic cloud with sufficient number of atoms available in the lower energy state for selective absorption and subsequent fluorescence emission. As the plasma cools, the number of atoms in the lower energy state will begin to increase with the reduction of the thermally excited population in the upper energy levels which contributes to LIBS emission, as seen in Figs. 3 and 5. When the excitation laser is in resonance with $\mathrm{Al}$ atoms in the lower energy state, the atoms are excited back to the upper energy level which results in LIF emission. The limit on the persistence of the LIF emission is primarily due to diffusion and reaction of the ambient species with the plume species (chemical reactions to form molecules) as well as a reduction in temperature.

An enhancement in the LIBS signal is evident at early times $(\widetilde{<30}-50 \mu \mathrm{s})$ when a partial pressure of 100 Torr $\mathrm{O}_{2}$ is present in the ambient environment, as shown in Fig. 5(a). Similarly, a pronounced LIF enhancement can be seen in Fig. 5(b) in the temporal window range $\sim 50-125 \mu$ s with the addition of 100 Torr $p_{o}$. The magnitude of both the LIBS and LIF enhancement is reduced with further increase of the oxygen partial pressure along with a reduction in persistence. These temporal features of LIBS and LIF can be explained by considering the inter-relation between plasma cooling due to expansion and thermodynamics of $\mathrm{AlO}$ formation. The reaction routes for $\mathrm{AlO}$ formation are exothermic, and hence the addition of $p_{o}$ will lead to a rise in temperature of the plasma along with depletion of $\mathrm{Al}$ atoms; these two processes may have opposing effects on the LIBS signal. Hence the increase in LIBS signal at early times could be due to the rise in temperature released during AlO formation, and the rapid decay in LIBS signal at later times could be due to depletion of the Al population in the plasma plumes. However, according to AlO thermodynamics and assuming LTE, AlO formation is favored when the plasma temperatures are in the range of $~ 3000-6000 \mathrm{~K}[35,44]$. With increasing time, because of plasma cooling, the temperature of the plasma becomes low enough to support AlO emission [40, 43]. This is consistent with observation of intense AlO emission in the $\sim 10-60 \mu$ s temporal window. Additionally, $\mathrm{Al}$ atoms in the lower energy state persist even at cooler temperatures and are therefore available for excitation by the probe laser at later times. This leads to enhancement in LIF at later times 
$(\sim 75 \mu \mathrm{s})$ in 100 Torr $p_{o}$. With the addition of further partial pressure of $\mathrm{O}_{2}$, the whole process occurs earlier with a reduction in intensity and persistence of both LIBS and LIF signals.

The measured $\mathrm{AlO}$ molecular temperature given in Fig. 7 is time-averaged $(10 \mu \mathrm{s}$ delay and $50 \mu \mathrm{s}$ integration time). Hence, the decrease in temperature as the $\mathrm{O}_{2}$ concentration is increased from 100 to 400 Torr $p_{o}$ is partly due to the majority of the energy being released by the oxidation of $\mathrm{Al}$ occurring at earlier times as the $\mathrm{O}_{2}$ concentration is increased; however, further time-resolved studies on $\mathrm{AlO}$ emission at various oxygen pressures are needed to confirm this. In the presence of $\mathrm{O}_{2}$ partial pressure the LIF emission persisted $\sim 200 \mu$ s, which is significantly higher than atomic or molecular LIBS emission which persisted $\sim 75 \mu \mathrm{s}$. It indicates that at later times, the plasma temperature has decreased to a point where thermal population of the excited states is not significant.

Plasma local conditions affect LIBS emission according to Eqn. 1 and it is well known that the nature of the ambient medium influences the plasma temperature and its evolution. Piehler et al. [7] investigated the temperature evolution of $\mathrm{Al}$ plasma under similar conditions used in this present study in nitrogen and oxygen ambient, and their results showed that hotter plasmas are formed in $\mathrm{N}_{2}$ in comparison with air or oxygen. The measured excitation temperatures were 7100 and $5800 \mathrm{~K}$ at $1 \mu$ s and dropped to 5800 and $4100 \mathrm{~K}$ at $10 \mu$ for $\mathrm{N}_{2}$ and air ambient, respectively [7]. It is possible that the enhanced persistence of LIBS in $\mathrm{N}_{2}$ ambient can be correlated to hotter conditions that exist within the plasma in comparison with plasmas generated with a partial pressure of $\mathrm{O}_{2}$ present. Surmick and Parigger [50] noticed that the temperature decay was much slower at later times of plasma evolution. In their experiment (Al plasma in air), the molecular temperatures were found to decrease from 5100 to $3850 \mathrm{~K}$ from 10 to $100 \mu$ s time delays. In their work, simulated $\mathrm{AlO}$ spectra were fit to the measured $\mathrm{AlO}$ emission spectra to calculate the molecular temperature, which does not exhibit significant selfabsorption. The differences in measured temperatures at $10 \mu \mathrm{s}$ in references $[7,50]$ can be correlated to differences in the laser intensities used.

As $\mathrm{N}_{2}$ is used as a makeup gas to maintain the ambient pressure when the $p_{o}$ is varied, it was of interest in this work to compare the LIBS and LIF results when $\mathrm{O}_{2}$ is present to that of when pure $\mathrm{N}_{2}$ is present as the ambient environment. As can be seen in Figs. 2 and 4, the LIBS and LIF persistence is greatly reduced when any amount of $\mathrm{O}_{2}$ is present in the ambient environment. The reduction in the persistence of LIBS and LIF when $\mathrm{O}_{2}$ is present can be partially explained by the removal of atomic Al from the plume through oxidation, which is supported by the continual decrease in the LIBS and LIF persistence as the concentration of $\mathrm{O}_{2}$ is increased from 100 to 400 Torr $p_{0}$, as shown in Fig. 4. From inspection of Figs. 3 and 5, it can be seen that the LIBS and LIF signals under an ambient $\mathrm{N}_{2}$ environment are not always more intense over the lifetime of the plasma compared to when $\mathrm{O}_{2}$ is present, but both signals are always longer lived for ambient $\mathrm{N}_{2}$. 
The intensity as well as linewidth of the $\mathrm{Al}$ transition decrease with increasing time after the onset of the LPP plume regardless of the ambient conditions. Stark broadening dominates at early times, especially $\leqq 10 \mu$ s. Spectral broadening can be used to infer electron density of the plasma. The Stark width parameters for the Al I $394.40 \mathrm{~nm}$ transition were obtained from Konjevic and Wiese [51]. The calculated electron density $\left(\mathrm{e}^{-} . \mathrm{cm}^{-3}\right)$ from the measured Lorentzian widths are $(1.07 \pm 0.11) \times 10^{16}$ and $(8.8 \pm 0.90) \times 10^{15}$ at $3 \mu$ s and $(4.30 \pm 0.44) \times 10^{15}$ and $(3.91 \pm 0.39) \times 10^{15}$ at $13 \mu$ s for $\mathrm{N}_{2}$ and 200 Torr $p_{o}$, respectively. The presence of self-absorption in the Al I resonance lines leads to a reduction in the accuracy of the measured emission linewidths; therefore, the plasma densities presented here are considered for qualitative comparison in this work. While significant changes were noticed in the reported magnitude and decay of plasma excitation temperatures with variation in the nature of the ambient gas [7], the measured electron density changes were marginal and indicate that plasma chemistry controls the plume temperature when it evolves.

As seen in Figures 2 and 4, the spectral lines in LPPs are broadened at early times due to the Stark effect. As time evolves, the Stark effect becomes negligible, especially after $\sim 10 \mu$ s for ns-LA plumes, in comparison with the instrumental linewidth for LIBS. LIF emission is capable of providing high spectral resolution in comparison with LIBS, as it occurs when the plasma is cooler and the measured linewidth is not dictated by instrumental broadening. In order to obtain the true LIF linewidth, the excitation spectrum should be recorded as well.

We recently demonstrated the use of 2D-FS in highly transient laser ablation plumes by measuring simultaneous absorption wavelength and emission intensity from coupled electronic transitions $[25,52]$. Such 2D-FS plots of $\mathrm{Al}$ plumes in pure $\mathrm{N}_{2}$ and with 200 Torr $p_{o}$ are given in Fig. 8(a\&c). Excitation spectra are shown in Fig. 8(b\&d), which were produced through a lineout at the center of the emission line in the 2DFS images shown in Fig. 8(a\&c). The measured LIF linewidths (FWHM) determined by fitting the center of the Al I $394.40 \mathrm{~nm}$ emission in the excitation spectra with a Voigt line shape are $\sim 6.7 \mathrm{pm}$ and $\sim 4.0 \mathrm{pm}$ for pure $\mathrm{N}_{2}$ and 200 Torr $p_{0}$, respectively. Typically, LIF excitation/absorption linewidths provide the intrinsic (physical) spectral linewidths of the transitions; however, the Al transitions used in this study are resonance lines and hence self-absorption may be present. If self-absorption is present, the measured excitation spectral linewidths from 2D-FS may not be accurate, and can lead to an artificially broadened excitation spectrum. The effect of self-absorption may be reduced for the 200 Torr $p_{0}$ case compared to the ambient $\mathrm{N}_{2}$ case due to oxidation of $\mathrm{Al}$, which leads to a reduction in the total atomic number density of $\mathrm{Al}$ within the plume at the late delay times used for the 2D-FS measurement. Broadening of the measured LIF linewidths at later times is due to Doppler broadening and van der Waals broadening. The corresponding kinetic temperature determined from the Gaussian component of the measured LIF linewidth is $4362 \pm 479 \mathrm{~K}$ for the 200 Torr $p_{o}$ case. The kinetic 
temperature is not reported for the ambient $\mathrm{N}_{2}$ case due to suspected self-absorption of the emission. The 2D-FS measurement is a time integrated and spatially averaged measurement, and thus the 2D-FS derived kinetic temperatures are as well. The molecular temperature derived from PGOPHER (with 3-sigma errors derived from the fit) for the 200 Torr $p_{o}$ case of $3850 \pm 85 \mathrm{~K}$ is in relatively good agreement with the kinetic temperature of $4362 \pm 479 \mathrm{~K}$ determined from the excitation spectrum. The small difference in the kinetic and molecular temperature can be due to the inhomogeneity of the plasma and the corresponding spatial dependence on where the molecular and atomic emission is observed.
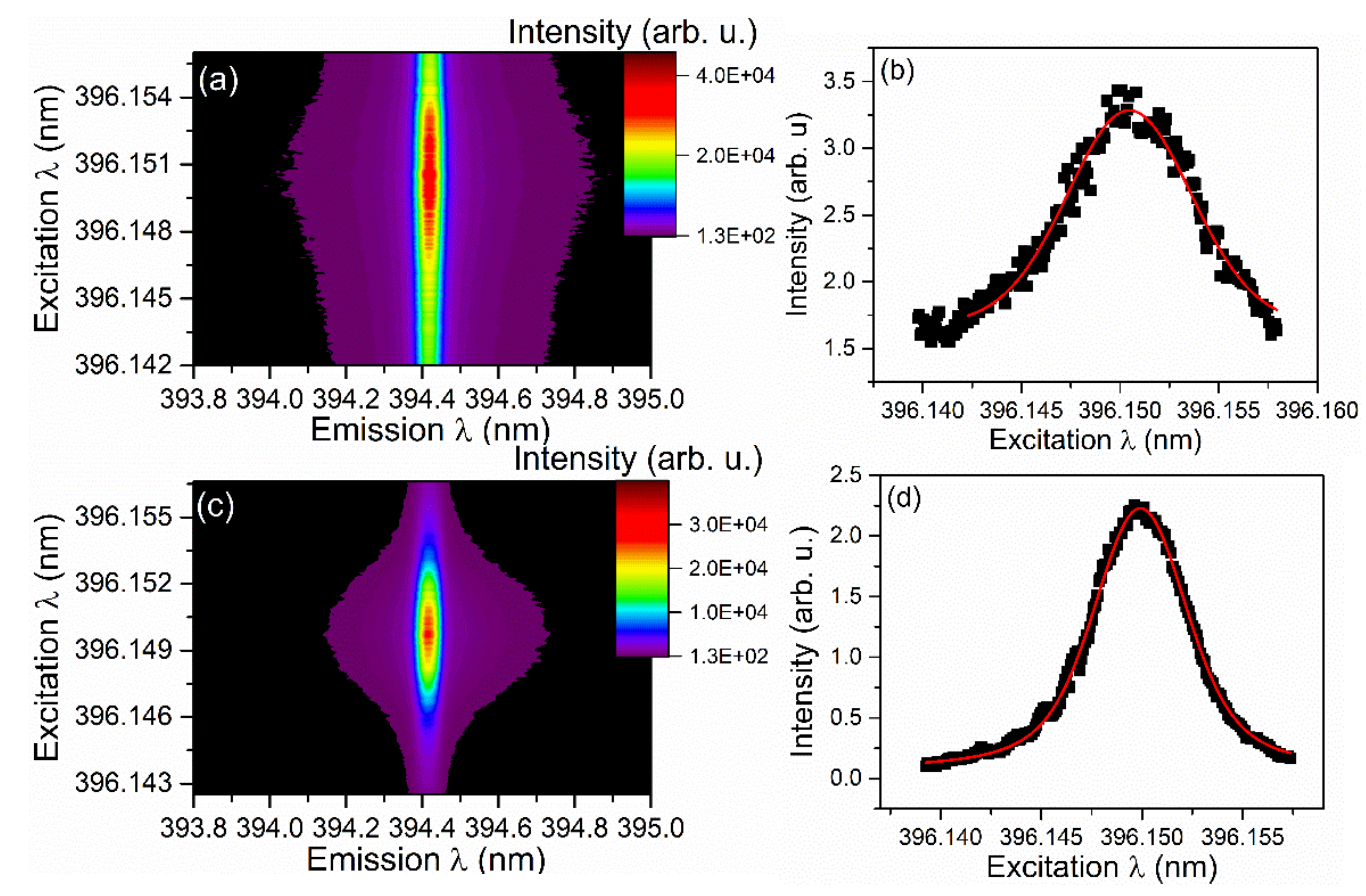

Figure 8. (a) 2D-FS image for ambient $\mathrm{N}_{2}$, (b) Voigt fit of excitation spectrum for ambient $\mathrm{N}_{2}$, (c) 2D-FS image for 200 Torr $p_{o}$, and (d) Voigt fit of excitation spectrum for 200 Torr $p_{o}$. LIF was collected over a gate width of $100 \mu \mathrm{s}$ and gate delay of $50 \mu \mathrm{s}$.

Oxygen-induced fluorescent state deactivation of organic molecules is well known [29]. However, limited studies exist on the role of oxygen in depletion of the total atomic number density and its effect on the measured LIF and LAS signals. Recently, Skrodzki et al. [27] compared the absorption signals of $\mathrm{U}$ species in air and $\mathrm{N}_{2}$ ambient and noticed significantly reduced ground state population persistence in air versus nitrogen environment. The recorded persistence for the lower energy state population of $\mathrm{U}$ I in $\mathrm{N}_{2}$ and air were $\sim 80 \mu$ s and $\sim 10 \mu$ s at 760 Torr, respectively. However, such an 8 -fold reduction in LIF persistence was not noticed in our present studies (factor of 2). Orsel et al. [53] recently evaluated the role of oxygen present in the background gas during the deposition of $\mathrm{YBiO}_{3}$ thin films by spatio-temporal mapping of the plasma constituents using LIF. Their results showed significant changes in spatial distributions of $\mathrm{Bi}, \mathrm{Y}$, and $\mathrm{YO}$ species in $\mathrm{Ar}$ and $\mathrm{O}_{2}$ environments, with the largest variation 
observed for YO. The LIF measurement in the present studies was carried out at a certain distance from the target surface $(\sim 1 \mathrm{~mm})$ and it may not be completely representative of the ground state population of the entire plume, considering the heterogeneous nature (time and space) of the laser ablation plumes.

Based on the present results on the role of $\mathrm{O}_{2}$ on excited and lower energy state population of an atomic transition, it can be concluded that the $\mathrm{O}_{2}$ is affecting the emission and fluorescence signal intensities and persistence greatly. However, the reduction in persistence depends strongly on plasma conditions and its expansion dynamics, target matrix, and chemical reaction pathways of the molecule of interest. $\mathrm{Al}$ and $\mathrm{U}$ metals are easily oxidized, though the depletion of atoms through oxidation is controlled by several factors which include molar concentration, rate of $\mathrm{O}_{2}$ diffusion into the plume, and shock wave evolution, for example. Given the sensitivity of these results to the ablation conditions, the comparisons here may be valid for metal targets, and significant differences could potentially be observed for non-metallic substrates.

\section{CONCLUSIONS}

In this article, a systematic study has been carried out to elucidate the effect that $\mathrm{O}_{2}$ in the ambient environment has on metal laser ablation plasmas. The LIBS and LIF emission spectra were reported for atmospheric pressure ambient environments where a range of $\mathrm{O}_{2}$ concentration was present $(100-400$ Torr $p_{o}$ ). Additionally, LIBS and LIF spectra under a pure $\mathrm{N}_{2}$ ambient environment were reported for comparison to measurements where $\mathrm{O}_{2}$ was present. Results clearly showed that the presence of oxygen in the ambient environment reduces the persistence of the LIBS and LIF emission. In comparison with ambient $\mathrm{N}_{2}$, both the LIBS and LIF persistence were decreased by a factor of $\sim 2$ with the addition of 100 Torr of oxygen, with further reduction in the persistence observed with increasing $p_{o}$.

The reduction of the LIF and LIBS persistence as the $\mathrm{O}_{2}$ concentration was increased from 100 to 400 Torr $p_{o}$ suggests that chemical reactions with $\mathrm{O}_{2}$ in the ambient environment depletes the $\mathrm{Al}$ population. AlO emission was found to be pronounced in the temporal window of $\sim 10-60 \mu \mathrm{s}$. However, both LIBS and LIF showed an enhancement in emission at early times $(\leq 50 \mu \mathrm{s}$ for LIBS and 50-130 $\mu \mathrm{s}$ for LIF) with the addition of partial pressure oxygen at lower $\mathrm{O}_{2}$ concentrations. The reaction routes for AlO formation are exothermic, and hence the addition of oxygen partial pressure will lead to a rise in temperature of the plasma. However, AlO formation is favored only in a certain range of temperatures $(\sim 3000-6000 \mathrm{~K})$ and at later times, because of the plasma cooling, $\mathrm{AlO}$ formation is reduced. $\mathrm{Al}$ atoms in the lower energy state persist even at cooler temperatures (compared to AlO formation), which are available for excitation by the probe laser at later times. This leads to enhancement in LIF at later times in

100 Torr $p_{o}$. With the addition of further partial pressure of $\mathrm{O}_{2}$, the whole process occurs earlier with reduction in intensity and persistence of both LIBS and LIF signals. 
Finally, it has to be highlighted that the reduction in persistence of LIBS and LIF in the presence of oxygen may depend strongly on plasma conditions, target matrix, oxidation properties, or thermodynamics various species in the plume. Hence, future work is necessary to elucidate the complex spatio-temporal evolution of the atomic and molecular emission within the plasma plume. Simultaneous absorption and emission measurements are suggested as future work to correlate the population of the ground state to the emission observed as a function of ambient environmental conditions. Temporallyresolved $\mathrm{AlO}$ emission studies should be performed as the concentration of oxygen in the ambient environment is varied to study the effect that increased oxygen concentration has on the rate of $\mathrm{AlO}$ formation. The results provided in this work are important for the LA absorption and emission communities where in situ analysis is desired and the presence of reactive species like $\mathrm{O}_{2}$ in the ambient environment are unavoidable.

\section{ACKNOWLEDGEMENTS}

This work is supported by the DOE/NNSA Office of Nonproliferation and Verification Research and Development (NA-22). Pacific Northwest National Laboratory is operated for the U.S. DOE by the Battelle Memorial Institute under Contract No. DEAC05-76RLO1830.

\section{REFERENCES}

[1] S. Musazzi, U. Perini, Laser-induced breakdown spectroscopy: Theory and Applications, Springer, Heidelburg, 2014.

[2] D.W. Hahn, N. Omenetto, Laser-Induced Breakdown Spectroscopy (LIBS), Part II: Review of Instrumental and Methodological Approaches to Material Analysis and Applications to Different Fields, Appl. Spectros., 66 (2012) 347-419.

[3] J.E. Barefield, E.J. Judge, K.R. Campbell, J.P. Colgan, D.P. Kilcrease, H.M. Johns, R.C. Wiens, R.E. McInroy, R.K. Martinez, S.M. Clegg, Analysis of geological materials containing uranium using laser induced breakdown spectroscopy, Spectrochim Acta B, 120 (2016) 1-8.

[4] X. Xiao, S. Le Berre, K.C. Hartig, A.T. Motta, I. Jovanovic, Surrogate measurement of chlorine concentration on steel surfaces by alkali element detection via laser-induced breakdown spectroscopy, Spectrochim. Acta, Part B, 130 (2017) 67-74.

[5] J.P. Singh, S.N. Thakur, Laser-induced breakdown spectroscopy, Elsevier, Amsterdam, 2007.

[6] R.E. Russo, X. Mao, H. Liu, J. Gonzalez, S.S. Mao, Laser ablation in analytical chemistry-a review, Talanta, 57 (2002) 425-451.

[7] T.N. Piehler, F.C. DeLucia, C.A. Munson, B.E. Homan, A.W. Miziolek, K.L. McNesby, Temporal evolution of the laser-induced breakdown spectroscopy spectrum of aluminum metal in different bath gases, Appl. Opt., 44 (2005) 3654-3660.

[8] D.W. Hahn, N. Omenetto, Laser-Induced Breakdown Spectroscopy (LIBS), Part I: Review of Basic Diagnostics and Plasma-Particle Interactions: Still-Challenging Issues Within the Analytical Plasma Community, Appl. Spectros., 64 (2010) 335a-366a.

[9] I. Ghebregziabher, K.C. Hartig, I. Jovanovic, Propagation distance-resolved characteristics of filamentinduced copper plasma, Opt. Exp., 24 (2016) 5263.

[10] P. Rohwetter, K. Stelmaszczyk, L. Woste, R. Ackermann, G. Mejean, E. Salmon, J. Kasparian, J. Yu, J.P. Wolf, Filament-induced remote surface ablation for long range laser-induced breakdown spectroscopy operation, Spectrochim Acta B, 60 (2005) 1025-1033. 
[11] E. Tognoni, G. Cristoforetti, Basic mechanisms of signal enhancement in ns double-pulse laserinduced breakdown spectroscopy in a gas environment, J. Anal. At. Spectrom., 29 (2014) 1318-1338.

[12] R.M. Measures, N. Drewell, H.S. Kwong, Atomic lifetime measurements obtained by the use of laser ablation and selective excitation spectroscopy, Physical Review A, 16 (1977) 1093-1097.

[13] C. Goueguel, S. Laville, F. Vidal, M. Sabsabi, M. Chaker, Investigation of resonance-enhanced laserinduced breakdown spectroscopy for analysis of aluminium alloys, J. Anal. At. Spectrom., 25 (2010) 635644.

[14] C. Dutouquet, J. Hermann, Laser-induced fluorescence probing during pulsed-laser ablation for three-dimensional number density mapping of plasma species, J Phys D Appl Phys, 34 (2001) 3356-3363.

[15] I.B. Gornushkin, S.A. Baker, B.W. Smith, J.D. Winefordner, Determination of lead in metallic reference materials by laser ablation combined with laser excited atomic fluorescence, Spectrochim Acta B, 52 (1997) 1653-1662.

[16] M. Miyabe, M. Oba, H. limura, K. Akaoka, A. Khumaeni, M. Kato, I. Wakaida, Ablation plume structure and dynamics in ambient gas observed by laser-induced fluorescence imaging spectroscopy, Spectrochim Acta B, 110 (2015) 101-117.

[17] K. Orsel, R. Groenen, H.M.J. Bastiaens, G. Koster, G. Rijnders, K.J. Boller, Spatial and temporal mapping of Al and AlO during oxidation in pulsed laser ablation of LaAlO3, J Instrum, 8 (2013) C10021.

[18] B.W. Smith, A. Quentmeier, M. Bolshov, K. Niemax, Measurement of uranium isotope ratios in solid samples using laser ablation and diode laser-excited atomic fluorescence spectrometry, Spectrochim Acta B, 54 (1999) 943-958.

[19] J. Li, Z. Hao, N. Zhao, R. Zhou, R. Yi, S. Tang, L. Guo, X. Li, X. Zeng, Y. Lu, Spatially selective excitation in laser-induced breakdown spectroscopy combined with laser-induced fluorescence, Opt. Express, 25 (2017) 4945-4951.

[20] R. Yi, J. Li, X. Yang, R. Zhou, H. Yu, Z. Hao, L. Guo, X. Li, X. Zeng, Y. Lu, Spectral Interference Elimination in Soil Analysis Using Laser-Induced Breakdown Spectroscopy Assisted by Laser-Induced Fluorescence, Anal. Chem., 89 (2017) 2334-2337.

[21] S.S. Harilal, N.L. LaHaye, M.C. Phillips, High-resolution spectroscopy of laser ablation plumes using laser-induced fluorescence, Opt. Express, 25 (2017) 2312-2326.

[22] K. Niemax, W. Sdorra, Optical-Emission Spectrometry and Laser-Induced Fluorescence of LaserProduced Sample Plumes, Appl. Opt., 29 (1990) 5000-5006.

[23] H.S. Kwong, R.M. Measures, Trace-Element Laser Microanalyzer with Freedom from Chemical Matrix Effect, Anal. Chem., 51 (1979) 428-432.

[24] S. Laville, C. Goueguel, H. Loudyi, F. Vidal, M. Chaker, M. Sabsabi, Laser-induced fluorescence detection of lead atoms in a laser-induced plasma: An experimental analytical optimization study, Spectrochimica Acta Part B: Atomic Spectroscopy, 64 (2009) 347-353.

[25] S.S. Harilal, N.L. LaHaye, M.C. Phillips, Two-dimensional fluorescence spectroscopy of laserproduced plasmas, Opt. Lett., 41 (2016) 3547-3550.

[26] S.S. Harilal, P.K. Diwakar, N.L. LaHaye, M.C. Phillips, Spatio-temporal evolution of uranium emission in laser-produced plasmas, Spectrochim. Acta B, 111 (2015) 1-7.

[27] P.J. Skrodzki, N. Shah, N. Taylor, K. Hartig, N. LaHaye, B. Brumfield, I. Jovanovich, M.C. Phillips, S.S. Harilal, Significance of plasma-ambient conditions in $U$ absorption and emission features in laser ablation plasmas, Spectrochim Acta B, 125 (2016) 112-119.

[28] Q.L. Ma, V. Motto-Ros, W.Q. Lei, M. Boueri, X.S. Bai, L.J. Zheng, H.P. Zeng, J. Yu, Temporal and spatial dynamics of laser-induced aluminum plasma in argon background at atmospheric pressure: Interplay with the ambient gas, Spectrochim Acta B, 65 (2010) 896-907.

[29] J.R. Lakowicz, Principles of Fluorescence Spectroscopy, 3rd ed., Springer, Heidelburg, 2006.

[30] S. Amoruso, R. Bruzzese, N. Spinelli, R. Velotta, Characterization of laser-ablation plasmas, J Phys Bat Mol Opt, 32 (1999) R131-R172. 
[31] X.K. Shen, Y.F. Lu, Detection of uranium in solids by using laser-induced breakdown spectroscopy combined with laser-induced fluorescence, Appl. Opt., 47 (2008) 1810-1815.

[32] B.W. Smith, I.B. Gornushkin, L.A. King, J.D. Winefordner, A laser ablation-atomic fluorescence technique for isotopically selective determination of lithium in solids, Spectrochim Acta B, 53 (1998) 1131-1138.

[33] A. Kramida, Y. Ralchenko, J. Reader, NIST ASD Team, NIST Atomic Spectra Database (ver. 5.3) [Online], in, National Institute of Standards and Technology, Gaithersburg, MD.

[34] K.C. Hartig, S.S. Harilal, M.C. Phillips, B.E. Brumfield, I. Jovanovic, Evolution of uranium monoxide in femtosecond laser-induced uranium plasmas, Opt. Exp., 25 (2017) 11477-11490.

[35] S.S. Harilal, B.E. Brumfield, B. Cannon, M.C. Phillips, Shock wave mediated plasma chemistry of AlO formation in laser ablation plumes Analytical Chemistry, 88 (2016) 2296-2302.

[36] M.A. Baig, M.A. Fareed, B. Rashid, R. Ali, On the Rydberg transitions and elemental compositions in the laser produced Al (6063) plasma, Phys Plasmas, 18 (2011) 083303.

[37] H.J. Kunze, Introduction to Plasma Spectroscopy, Springer, Heidelburg, 2009.

[38] J. Serrano, J. Moros, J.J. Laserna, Molecular signatures in femtosecond laser-induced organic plasmas: comparison with nanosecond laser ablation, Phys. Chem. Chem. Phys., 18 (2016) 2398-2408.

[39] J. Serrano, J. Moros, J.J. Laserna, Exploring the formation routes of diatomic hydrogenated radicals using femtosecond laser-induced breakdown spectroscopy of deuterated molecular solids, J. Anal. At. Spectrom., 30 (2015) 2343-2352.

[40] NIST-JANAF Thermochemical Tables, in http://kinetics.nist.gov/janaf/

[41] J.M. Peuker, P. Lynch, H. Krier, N. Glumac, On AlO Emission Spectroscopy as a Diagnostic in Energetic Materials Testing, Propell Explos Pyrot, 38 (2013) 577-585.

[42] S.S. Harilal, G.V. Miloshevsky, P.K. Diwakar, N.L. LaHaye, A. Hassanein, Experimental and computational study of complex shockwave dynamics in laser ablation plumes in argon atmosphere, Phys Plasmas, 19 (2012) 083504.

[43] S. Yuasa, Y.X. Zhu, S. Sogo, Ignition and combustion of aluminum in oxygen/nitrogen mixture streams, Combust Flame, 108 (1997) 387-396.

[44] X.S. Bai, V. Motto-Ros, W.Q. Lei, L.J. Zheng, J. Yu, Experimental determination of the temperature range of AlO molecular emission in laser-induced aluminum plasma in air, Spectrochim Acta B, 99 (2014) 193-200.

[45] G.R. Hébert, R.W. Nicholls, C. Linton, Absolute band strengths for the AlO blue-green (B2 $\sigma-X 2 \sigma)$ band system, Journal of Quantitative Spectroscopy and Radiative Transfer, 23 (1980) 229-235.

[46] M.D. Saksena, M.N. Deo, K. Sunanda, S.H. Behere, C.T. Londhe, Fourier transform spectral study of B2 $\Sigma+-X 2 \Sigma+$ system of AlO, J. Mol. Spectrosc., 247 (2008) 47-56.

[47] S.S. Harilal, C.V. Bindhu, M.S. Tillack, F. Najmabadi, A.C. Gaeris, Internal structure and expansion dynamics of laser ablation plumes into ambient gases, Journal of Applied Physics, 93 (2003) 2380-2388.

[48] S.G. Buckley, Laser-induced breakdown spectroscopy for toxic metal emission measurements: Experimental considerations and oxygen quenching, Environ Eng Sci, 22 (2005) 195-204.

[49] R.L. Gleason, D.W. Hahn, The effects of oxygen on the detection of mercury using laser-induced breakdown spectroscopy, Spectrochim Acta B, 56 (2001) 419-430.

[50] D.M. Surmick, C.G. Parigger, Time-resolved aluminium laser-induced plasma temperature measurements, J Phys Conf Ser, 548 (2014) 012046.

[51] N. Konjevic, W.L. Wiese, Experimental Stark Widths and Shifts for Spectral-Lines of Neutral and Ionized Atoms (a Critical-Review of Selected Data for the Period 1983 through 1988), J Phys Chem Ref Data, 19 (1990) 1307-1385.

[52] M.C. Phillips, B.E. Brumfield, N. LaHaye, S.S. Harilal, K.C. Hartig, I. Jovanovic, Two-dimensional fluorescence spectroscopy of uranium isotopes in femtosecond laser ablation plumes, Scientific Reports, 7 (2017) 3784. 
[53] K. Orsel, R. Groenen, B. Bastiaens, G. Koster, G. Rijnders, K.J. Boller, Laser-induced fluorescence analysis of plasmas for epitaxial growth of YBiO3 films with pulsed laser deposition, Apl Mater, 4 (2016) 126102. 

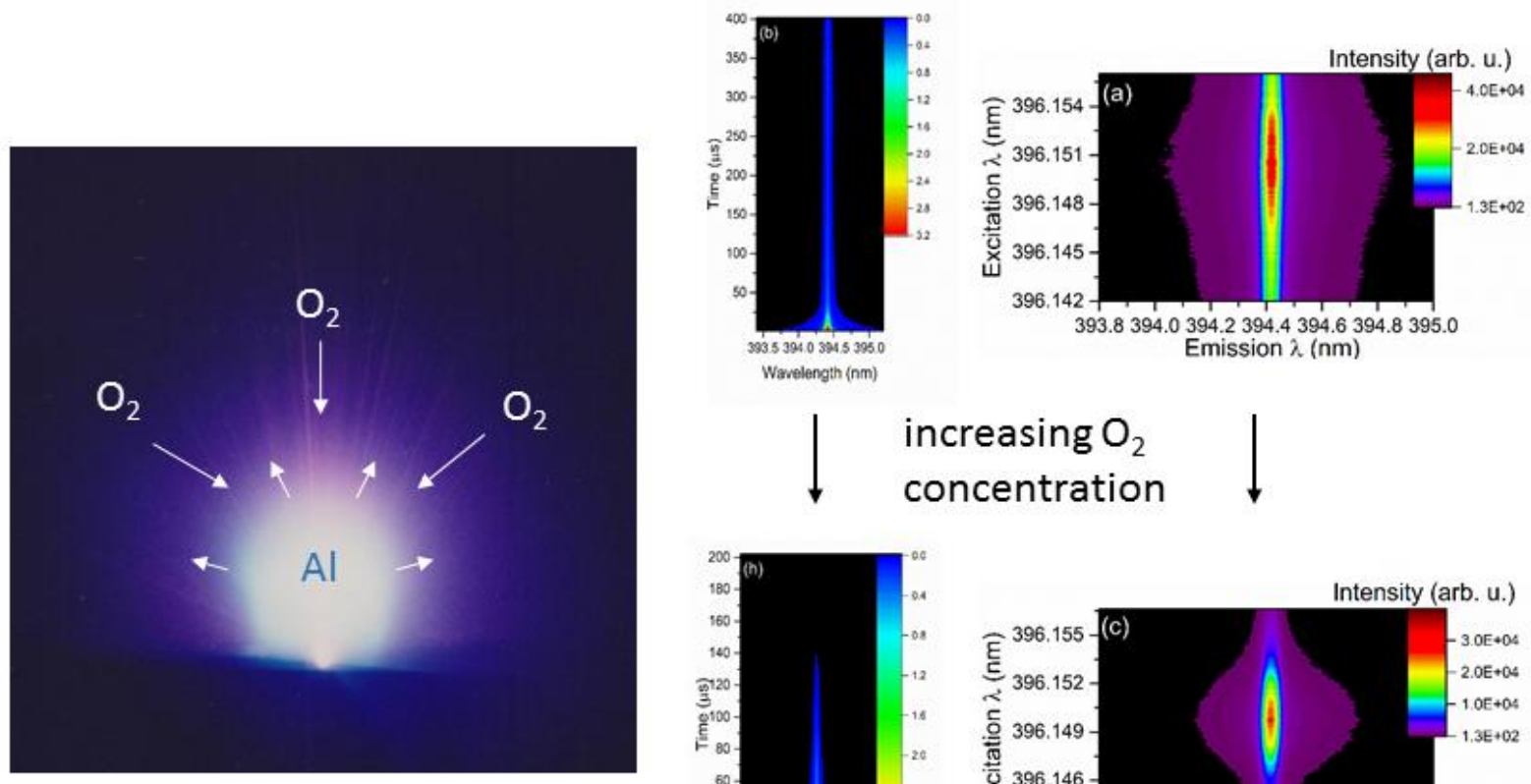

elength $(\mathrm{nm})$

increasing $\mathrm{O}_{2}$

concentration
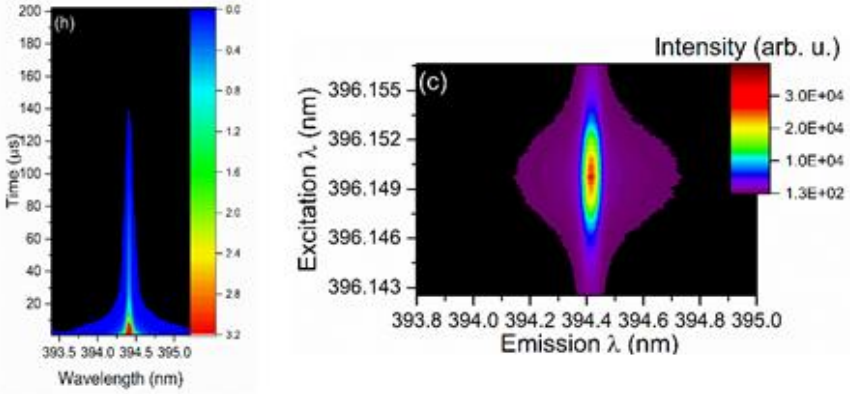